\title{
Grazing angle X-ray fluorescence from periodic structures on silicon and silica surfaces ${ }^{2}$
}

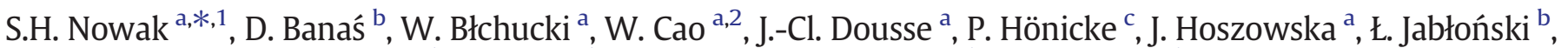 \\ Y. Kayser ${ }^{\text {a,3 }}$, A. Kubala-Kukuś ${ }^{\text {b }}$, M. Pajek ${ }^{\text {b }}$, F. Reinhardt ${ }^{\text {c }}$, A.V. Savu ${ }^{\text {d }}$, J. Szlachetko b,e \\ a Physics Department, University of Fribourg, $\mathrm{CH}-1700$ Fribourg, Switzerland \\ ${ }^{\mathrm{b}}$ Institute of Physics, Jan Kochanowski University, 25-406 Kielce, Poland \\ c Physikalisch-Technische Bundesanstalt (PTB), D-10587 Berlin, Germany \\ d Microsystems Laboratory (LMIS1), Ecole Polytechnique Fédérale de Lausanne, CH-1015 Lausanne, Switzerland \\ e Paul Scherrer Institut, CH-5232 Villigen PSI, Switzerland
}

Various 3-dimensional nano-scaled periodic structures with different configurations and periods deposited on the surface of silicon and silica substrates were investigated by means of the grazing incidence and grazing emission X-ray fluorescence techniques. Apart from the characteristics which are typical for particle- and layer-like samples, the measured angular intensity profiles show additional periodicity-related features. The latter could be explained by a novel theoretical approach based on simple geometrical optics (GO) considerations. The new GO-based calculations were found to yield results in good agreement with experiment, also in cases where other theoretical approaches are not valid, e.g., periodic particle distributions with an increased surface coverage.

\section{Introduction}

In the last years a variety of 3-dimensional nano-scaled structures featuring island-like morphologies or high surface roughnesses were investigated by means of grazing incidence (GIXRF) and grazing emission (GEXRF) X-ray fluorescence measurements. Because such nanosystems exhibit unusual spectral features [1,2] that are difficult or even impossible to reproduce with presently available numerical models [3,4], a novel approach employing geometrical optics (GO) calculations was developed [5].

A particular aim of the GO simulations was to investigate the changes in the intensity dependence of the X-ray fluorescence (XRF) on the grazing angle when the distribution of the nano-sized particles on the sample surface evolves from dispersed particles with a low surface coverage to a continuous layer structure. As a model we used an easy to parameterize system consisting of periodically distributed identical brick-like elements. Such a system can be described with only three

\footnotetext{
Selected paper from the 15th International Conference on Total Reflection X-ray Fluorescence Analysis and Related Methods, and the 49th Annual Conference on X-ray Chemical Analysis (TXRF2013), Osaka, Japan, 23-27 Sept. 2013.

* Corresponding author.

E-mail address: nowak@ifg-adlershof.de (S.H. Nowak).

1 Present address: IfG - Institute for Scientific Instruments GmbH, Berlin, Germany.

2 Present address: Physics Department, University of Oulu, FI-90014 Oulu, Finland.

3 Present address: Paul Scherrer Institut, CH-5232 Villigen PSI, Switzerland.
}

parameters, the period $p$ of the pattern and the width $l$ and height $h$ of the individual bricks. From the experimental results concerning samples with dense particle distributions [5-7] smooth transitions from particle-like to layer-like structures were expected. In contrast to that, the GO simulations showed very sharp and intense peaks, whose positions were found to vary strongly as a function of the height and period of the pattern. In addition, as a result of constructive or destructive interference effects, the peak intensities appeared to be very sensitive to the alteration of the bricks' width.

The above mentioned peaks which, to our best knowledge, were never observed experimentally before cannot be described in terms of standard diffraction patterns. They can neither be explained by existing theoretical models. Actually, GIXRF investigations of periodic surface structures were already reported by Tsuji et al. [2]. However, comparing the angular profiles obtained in Tsuji's work with those corresponding to smooth surfaces, one sees that only one additional peak is observed near the critical angle in the angular profiles of the periodic samples. In theory, a study describing the GEXRF from a grating-like substrate was published [8]. In this work, it is predicted that, as a result of the diffraction on the grating surface, distinct peaks should be observed in the profile representing the angular dependence of the fluorescence signal. According to this model the magnitude of these peaks could exceed several times the X-ray intensity compared to a flat substrate under the same conditions of excitation. However, due to the very small wavelengths corresponding to X-rays, one can expect such diffraction peaks to be observable only for soft X-rays and very dense $(p<1 \mu \mathrm{m})$ gratings. 


\section{Grazing angle geometries}

Total reflection X-ray fluorescence (TXRF) analysis and its derived methods GIXRF and GEXRF represent powerful tools for the analysis of surfaces [9-15]. In particular they are very useful for trace elements' analysis $[6,16]$, characterization of thin layers $[7,17]$ and depth profiling [18-20]. The usefulness of grazing XRF techniques for nanoparticle characterization has also been demonstrated [8,21-26]. Furthermore, like other XRF-based methods, these techniques are non-destructive and offer elemental sensitivity [9]. If a crystal spectrometer, which offers high-energy-resolution detection, is used even different compounds of the same element can be distinguished [9].

\subsection{Total reflection X-ray fluorescence}

In TXRF [10-12] the incident radiation is impinging on the sample at an angle set below the critical angle of total external reflection. Above the sample surface an X-ray Standing Wave (XSW) field arises due to the interferences between the incident and reflected beams. Therefore, only the surface and the region lying a few nanometers below it are excited. The fluorescence radiation is measured by a detector positioned perpendicularly to the sample surface at a very close distance, which ensures a large solid angle for the detection of the sample fluorescence. In addition, due to the low penetration depth of the totally-reflected incident photon beam, the method benefits from the substantial decrease of the background radiation originating from the substrate material.

The TXRF technique is mainly used for micro- and trace-element analysis of evaporated solvents, aerosols and powders [27,28]. Another common application is the control of surfaces' contamination [29]. The detection limits are of the order of picograms or even femtograms if pre-concentration methods are applied [11].

\subsection{Grazing incidence X-ray fluorescence}

The GIXRF technique $[10-12,30]$ is an extension of TXRF in which the X-ray fluorescence is measured as a function of the angle between the sample surface and the incident beam. The angle is varied continuously from $0^{\circ}$ to far above the critical angle of total external reflection.

The intensity of the XSW field above the sample surface and the penetration depth into the substrate depend pronouncedly on the incidence angle. This provides access to the depth dependent elemental concentration distributions in the near-surface region. For grazing angles below the critical angle the beam is reflected at almost $100 \%$, thus only the sample surface and the first few nanometers below the surface are excited. The excitation of the near-surface region depends on the strength of the XSW field and the penetration into the sample is accounted for the evanescent field. On the contrary, for incidence angles above the critical angle, the XSW field above the surface almost vanishes and the penetration depth, which depends on the absorption of the radiation by the sample material, is maximized. Near the critical angle of total external reflection the XSW field maximum is at the sample surface and the penetration depth into the sample changes drastically.

Since by tuning the incidence angle both the XSW field strength and penetration depth can be varied, GIXRF is well suited for depth profiling measurements [31] as well as for the characterization of thin layers and on-surface micro-sized objects [32].

\subsection{Grazing emission X-ray fluorescence}

\subsubsection{Standard GEXRF}

In GEXRF the fluorescence radiation is detected at exit angles around the critical angle [7,12-15,33-35]. GEXRF can be regarded as an inverted GIXRF experiment where the X-ray source and detector are exchanged (see Fig. 1). Thus, according to the reciprocity theorem [36], the theoretical description of GEXRF can be based on derivations made for TXRF and GIXRF [33,37]. However, more formal approaches employing the Maxwell's equations $[13,35]$ and field expansions over plane waves $[8,21]$ are also found in the literature.

The dependence of GEXRF on the exit angle is analogous to that of GIXRF on the incidence angle. For exit angles below the critical angle of total external reflection, only the first few nanometers below the surface do indeed contribute to the measured fluorescence intensity. For exit angles above the critical angle, the detection sensitivity for X-rays emitted from deeper parts of the sample increases. In the latter case the accessible depth region is limited by the self-absorption of the fluorescence X-rays. Furthermore, the interference of the fluorescence $\mathrm{X}$-rays themselves affects the detected XRF intensity similarly to the XSW field variation in the case of GIXRF.

Contrary to GIXRF, the GEXRF setup allows performing microanalysis and surface mapping provided that an intense enough X-ray microbeam is used [16]. A further advantage of the GEXRF method as compared to GIXRF is that the X-ray fluorescence can be produced by any type of ionizing radiation, e.g., photon-, electron-, proton- and ion-beams [38].

On the other hand, the main drawback of the GEXRF setup resides in the lack of tunability of the critical angle. In GIXRF the latter can be modified by tuning the energy of the incident radiation. This is not possible for GEXRF since the energy of the fluorescence radiation corresponds to the characteristic X-ray emission of the elements contained in the sample.

\subsubsection{High-resolution GEXRF}

A further advantage of the GEXRF geometry is the possibility of combining it with wavelength-dispersive instruments for the detection of the fluorescence radiation $[6,7,16,39]$. This provides a much higher spectral resolution and thus a higher chemical sensitivity. Note that a good separation of the fluorescence lines is particularly important for the K-shell X-ray emission of samples made of light elements [6].

In high-resolution GEXRF the grazing emission conditions are achieved by turning the sample surface close to the emission direction defined by the Bragg angle of diffraction $\Theta_{B}$. Since, for a given wavelength and the diffraction order, only the X-rays hitting the crystal surface with the angle $\Theta_{B}$ are diffracted towards the detector, the crystal automatically selects a quasi-parallel incident beam, the tiny divergence of the beam being determined by the Darwin width (if perfect crystals are used) or the mosaicity width (for mosaic crystals). As a consequence the slit system used in the standard GEXRF geometry to define the exit angle with a sufficient angular resolution is no longer needed (see Fig. 2). In addition, the slit-less geometry employed in the high-
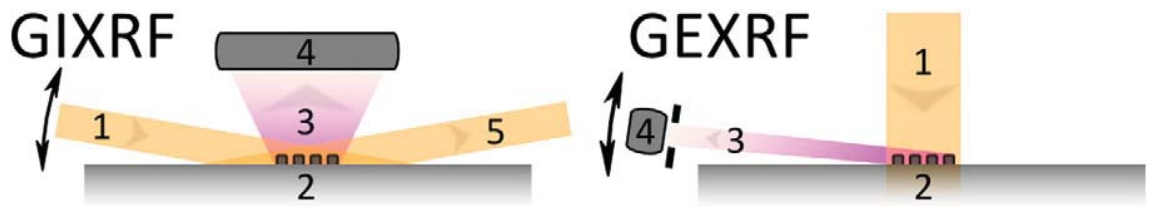

Fig. 1. Comparison of the GIXRF and GEXRF geometries. 1. Incident beam, 2. sample, 3. detected fluorescent radiation, 4. detector, 5. reflected beam. In the GIXRF setup the incident X-ray radiation is collimated, the angle of incidence is tunable and the fluorescent radiation is detected perpendicularly to the sample surface within a large solid angle. In the GEXRF setup the fluorescent radiation is detected within a small solid angle, the angle of detection is tunable and the incidence angle is large, e.g., $90^{\circ}$. In the case of GEXRF the excitation can be produced by any kind of ionizing radiation (X-ray radiation, electron beam, ion beam) 


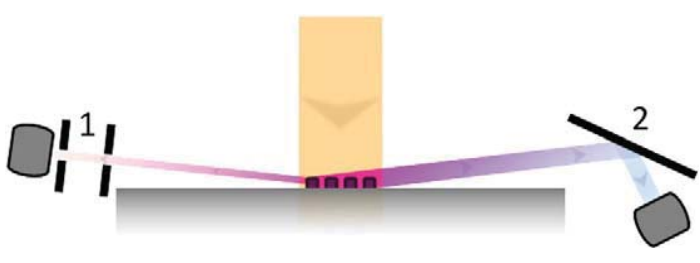

Fig. 2. Comparison of the standard (left) and high-resolution (right) GEXRF geometries. 1. Collimating slits, 2. Bragg crystal. In the standard GEXRF setup the solid angle of detection is small due to the use of collimating slits. In the high-resolution geometry the fluorescence X-ray beam is automatically collimated by the crystal via the Bragg law, which allows a slit-less operation.

resolution GEXRF leads to an increase of the fluorescence signal in the detector since the whole irradiated surface of the sample contributes to the measured intensity.

GEXRF setups based on wavelength-dispersive detectors present the additional advantage of having an excellent background rejection capability. Most of the background events produced in the sample have indeed a different energy than the one of the fluorescence X-rays of interest. As a consequence, they are not diffracted by the crystal and are thus eliminated. The remaining background which is related to scattered photons, cosmic rays and electronic noise of the detector can be minimized by means of energy discrimination algorithms [40].

\subsection{Main types of angular intensity profiles}

Depending on the sample's morphology three well described types of GEXRF and GIXRF angular profiles are distinguished: bulk-like, layer-like and particle-like [10,34,35] (see Fig. 3). For bulk structures the radiation is refracted once at the vacuum-bulk interface and the angular fluorescence profile follows the angle dependent penetration depth of the corresponding X-ray radiation. Also the angular profile of a layer-like structure conforms to the penetration depth dependence until the latter reaches the value of the layer thickness. Above that point an interference pattern can be observed in the angle dependent

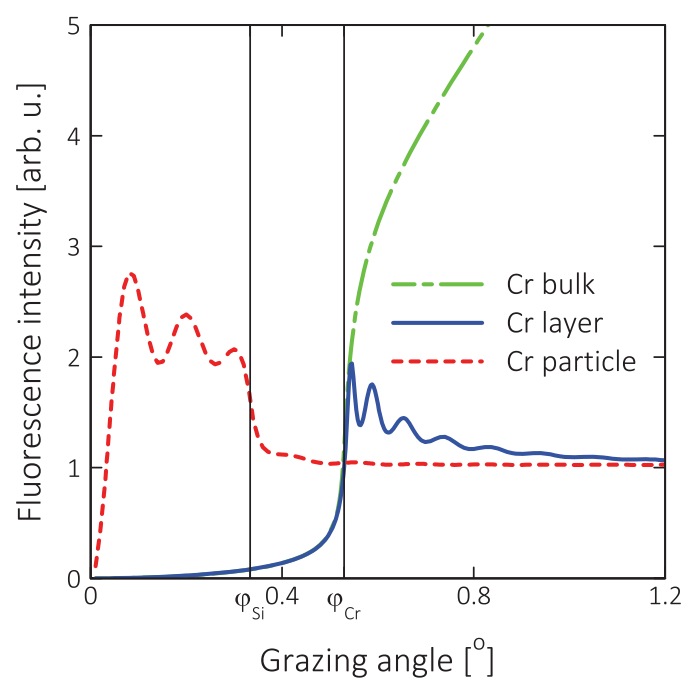

Fig. 3. Bulk-like, layer-like and particle-like GEXRF angular profiles calculated for the $\mathrm{Cr} \mathrm{K} \alpha$ fluorescence from: a $\mathrm{Cr}$ bulk sample (green dash-dotted line), a $50 \mathrm{~nm}$ thick $\mathrm{Cr}$ layer on a Si substrate (blue line), and a $50 \mathrm{~nm}$ high $\mathrm{Cr}$ particle lying on a Si substrate (red dashed line). In both the layer-like and particle-like profiles interference patterns are visible. The bulk and layer profiles coincide below the critical angle for $\mathrm{Cr}\left(\varphi_{\mathrm{Cr}}\right)$. This is due to the evanescent X-ray radiation that decays within the layer depth. Because the inflection points of the layer-like and particle-like profiles are given by the critical angles for $\operatorname{Si}\left(\varphi_{\mathrm{Si}}\right)$ and $\mathrm{Cr}\left(\varphi_{\mathrm{Cr}}\right)$, respectively, which differ by about $0.2^{\circ}$, the signals from the two structures can be easily separated. GIXRF angular profiles present similar features. fluorescence intensity. In GIXRF, respectively GEXRF this interference pattern can be explained by multiple reflections of the incident, respectively the emitted X-ray photons on the top and bottom interfaces of the layer [37]. In contrast, the angle dependent fluorescence intensity of particle-like structures shows strong intensity increase and modulations below the critical angle [22-24]. In GEXRF these oscillations are due to interferences between the fluorescence X-rays following different exit paths [21], while in GIXRF these oscillations originate from interferences between the incident and reflected X-rays, i.e., from intensity changes in the XSW field [24].

From the angular intensity profiles various information on the sample structure can be retrieved. For both bulk-like and layer-like profiles the density of the material can be deduced from the position of the critical angle. For nanoscaled particle- and layer-like structures the absorption is negligibly small. In this case the number of detected fluorescence photons is thus directly proportional to the number of excited sample atoms. The interference pattern of the layer-like structures can be inspected to determine the layer thickness. Similarly valuable information can be deduced from the particle-like interference patterns $[21,24]$. However, as shown in Ref. [24], the nonuniform particle size distribution and the limited coherence length of X-rays significantly reduce the interferences' amplitude.

\subsection{Angular profiles of dense particle distributions}

The angular profiles of the X-ray fluorescence emitted by surfaces characterized by dense particle distributions are usually treated as profiles of rough layer-like surfaces. The roughness can be introduced as a small perturbation of the interface potential within the Nevot-Croce model [1,41], a stack of layers with reduced average densities [41,42] or as a superposition of layers of different thicknesses [6,43]. A slightly different approach was presented in Ref. [44] where, in order to calculate the influence of the absorption effects, the sample surface was considered to be made of a series of small towers of variable height and width.

While being certainly useful, the above mentioned models appear to be too simple to correctly describe dense particles' distributions because effects related to highly correlated height distributions and large roughnesses [1] or to the propagation of the X-ray radiation through several consecutive particles [2] are not considered. The same holds for periodic surface structures whose angular intensity profiles cannot be predicted properly by any of these models. In particular, the characteristic features related to the structure periodicity are not reproduced in the simulated angular profiles.

\section{Geometrical optics approach}

\subsection{Motivation and basic principles}

A correct interpretation of the angular profiles of intermediate structures is of prime importance, in particular for investigation of nanostructures and for TXRF measurements of droplets where the quantification problem is one of the main issues [45].

Recently a novel approach to GIXRF/GEXRF simulations, employing geometrical optics (GO) calculations, was proposed [5]. The GO model traces the photon paths taking into account reflection, refraction, transmission and absorption effects. The phase shift due to different possible paths and the probability for each photon to interfere with itself are calculated. In order to obtain information on the whole sample, the X-ray tracing is calculated for many points distributed randomly throughout the sample.

To reduce the complexity of the algorithm two assumptions are made (1) the incident (GIXRF) or exiting (GEXRF) X-ray beam is perfectly parallel; (2) the investigated structure has only horizontal and vertical interfaces. In a 2-dimensional coordinate system, where the $x$ axis lies in the plane of the substrate surface and the $y$ axis is 
perpendicular to that surface, reflections at the horizontal interfaces change only the $y$-component of the wave vector, the length of the latter remaining, however, unchanged. At vertical interfaces the reflections can be neglected since in GIXRF or GEXRF measurements the incidence angles for vertical interfaces are large so that the X-ray reflectivity is negligibly small.

X-rays can be treated within the GO approximation provided that their wavelength is smaller than the dimensions of the investigated structures. For X-rays with photon energies above $1 \mathrm{keV}(\lambda<1.2 \mathrm{~nm})$ this is usually the case. In the GO approach specific geometrical boundary conditions can be taken into account.

\subsection{Intensity peak positions}

For periodic surface patterns, like in the case of layer-like samples, $\mathrm{X}$-rays can be reflected many times between the top and bottom interfaces. This happens if the distance between two consecutive reflections is a fraction or a multiple of the structure period (see Fig. 4). Assuming that the ray path is not refracted at the side interfaces of the structure, this condition can be written as follows:

$\tan \phi_{M}=M \frac{h}{p}, \quad M \in \mathbb{Q}_{+}$

where $h$ and $p$ are the height and period of the pattern, respectively, and $\phi_{M}$ is the angle between the X-ray path in the structure's material and the direction parallel to the interface.

If the refraction between the vacuum and structure's material takes place on the side interface the grazing angle $\varphi$ can be approximated by $\phi$, whereas in the case that the refraction takes place on the top interface the relation between $\varphi$ and $\phi$ is more complex. It can be, however, calculated using the incident and refracted wave vectors:

$\mathrm{k}=k(\cos \varphi, \sin \varphi)$

$\mathrm{k}^{\prime}=k\left(\cos \varphi, \sqrt{n^{2}-\cos ^{2} \varphi}\right)$

where $k$ is the wave vector length and $n$ the complex refraction index of the material. Using then the relations

$\tan \varphi=\frac{\sin \varphi}{\cos \varphi}$

$\tan \phi=\frac{\operatorname{Re}\left(\sqrt{n^{2}-\cos ^{2}(\varphi)}\right)}{\cos \varphi}$, one obtains an implicit relation:

$\tan \varphi=\frac{\sin \varphi}{\operatorname{Re}\left(\sqrt{n^{2}-\cos ^{2}(\varphi)}\right)} \tan \phi$,

which can be easily solved numerically.

The fact that multiple reflections occur only for distinct grazing angles (parameterized by $M$ ) gives rise to sharp peaks in the calculated angular profiles. However, not all predicted peaks can be observed because the intensities of some of them are strongly diminished by destructive interferences.

\section{Experiments}

\subsection{Samples}

In order to evaluate the GO approach and compare the GIXRF and GEXRF results, several sets of samples with different periodic onsurface nanostructures were fabricated. The structures included pads, stripes, wedges and disks (see Fig. 5) and were made of $\mathrm{Cr}, \mathrm{Ni}$, and $\mathrm{Co}$. Silicon and silica were chosen as substrate materials. The latter are characterized by a small decrement factor in the refractive index, thus relatively small critical angles. In contrast, the decrement factor in the refractive index of $3 d$ transition metals is, in comparison, relatively large. As a result the critical angle for total reflection of the structures' material is significantly different from that of the substrate. Consequently, the combination of $3 d$ transition metals deposited on low-Z substrates allows a clear separation of particle-like and layer-like components in the angular intensity profiles (see Fig. 4).

\subsection{GIXRF measurements}

The GIXRF measurements were performed in the laboratory of the Physikalisch-Technische Bundesanstalt (PTB) at the electron storage ring BESSY II [46]. The samples were installed in the ultra-high vacuum chamber developed recently at the PTB [47]. This chamber is equipped with a multi-axis manipulator comprising a $x-y-z$-sample stage placed on a $\chi-\varphi-\vartheta$-table. Here $\chi$ denotes the angle between the polarization plane of the incident synchrotron beam and the sample surface. For $\chi=90^{\circ}$ the scattering radiation produced in the sample is minimized, whereas for $\chi=0^{\circ} s$-polarized measurements can be performed. If $\chi=90^{\circ}$, the angle between the incident beam and the substrate surface is given by $\varphi$. With this target chamber, GIXRF and TXRF as well as conventional $45^{\circ} / 45^{\circ} \mathrm{XRF}$ measurements are feasible. Note that the sample can also be rotated around the normal vector of the sample surface by means of the $\vartheta$-stage.

The centers of the measured structures were aligned on the rotation axes of the $\vartheta$ and $\varphi$ stages. The measurements were performed for

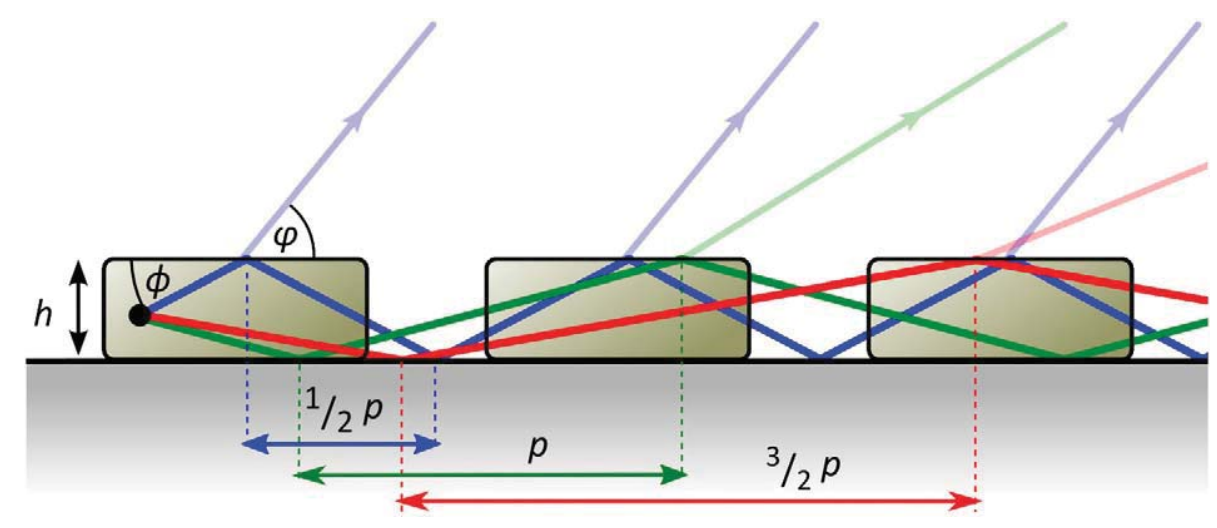

Fig. 4. Multiple reflections in a periodic structure. 


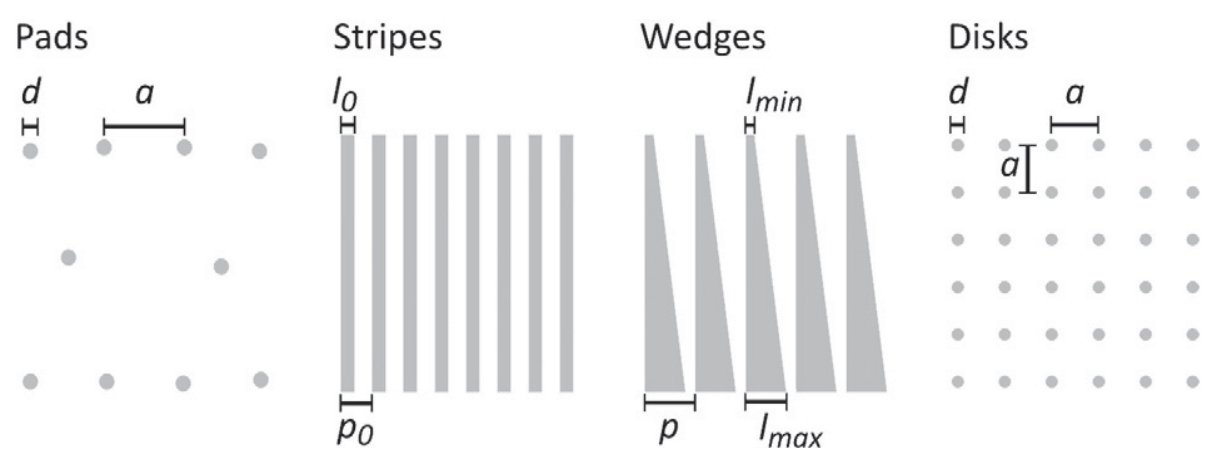

Fig. 5. Schematic drawings of different sample structures. $d$ is the diameter of a structure element, $a$ the smallest distance between neighboring elements, $l$ the width of a structure element and $p$ the structure period.

different tilt angles $\vartheta$ of the structures with respect to the plane of incidence. At each $\vartheta$-position the fluorescence photons were recorded for a series of incidence angles $\varphi$ using a silicon drift detector (SDD). The GIXRF angular intensity profiles were normalized with the numbers of incident photons which were measured with a thin transmission diode. The measured fluorescence intensities were then corrected for the known efficiency and dead time of the detector as well as for the angle dependent effective solid angle of detection [48].

\subsection{High-resolution GEXRF measurements}

The high-resolution (GEXRF) measurements were performed at the beamline ID21 of the European Synchrotron Radiation Facility (ESRF) in Grenoble, France, and at the SuperXAS beamline of the Swiss Light Source (SLS) of the Paul Scherrer Institute (PSI) in Villigen, Switzerland. For both series of measurements, the von Hamos curved crystal X-ray spectrometer of the University of Fribourg [49] was used.

At the ESRF the synchrotron radiation was produced by two undulators mounted in series. Upper-harmonics photons were rejected by means of a Ni coated mirror set at a cut-off angle of $7.5 \mathrm{mrad}$. The beam energy was tuned to $6.48 \mathrm{keV}$ by means of a double $\mathrm{NiB}_{4} \mathrm{C}$ multilayer monochromator. In order to define the irradiated sample area vertical and horizontal slits with adjustable widths were used to define the beam size. The latter varied between $0.05 \times 0.2 \mathrm{~mm}^{2}$ and $1.2 \times 1.2 \mathrm{~mm}^{2}$. For the biggest beam size the resulting intensity on the samples was of the order of $2.8 \cdot 10^{13}$ photons/s.

For the measurements performed at the SLS, a $2.9 \mathrm{~T}$ bending magnet was employed for the production of the synchrotron radiation. The beam energy was tuned to $8.5 \mathrm{keV}$ using a Rh coated double crystal monochromator. In order to define the beam size a height of $0.5 \mathrm{~mm}$ was adopted for the horizontal slit, whereas the width of the vertical slit was varied between 0.2 and $3.5 \mathrm{~mm}$. For the largest slit aperture the intensity on the sample was in the range of $7 \cdot 10^{11}$ photons $/ \mathrm{s}$.

The GEXRF angular profiles were measured using the K $\alpha \mathrm{X}$-ray lines of $\mathrm{Cr}, \mathrm{Co}$ and $\mathrm{Ni}$. For the $\mathrm{Cr}$ measurements the von Hamos spectrometer was equipped with a $\mathrm{SiO}_{2}(1 \overline{1} 10)$ crystal, the fluorescence X-ray line being observed in the second order of diffraction. For the Co and $\mathrm{Ni}$

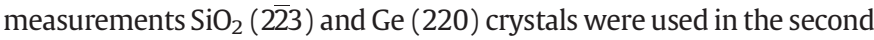
and first orders of diffraction, respectively.

\subsubsection{Target alignment}

For the GEXRF measurements a precise control of the exit angle $\varphi$, i.e., the angle between the sample surface and the direction of the emitted fluorescence X-rays which is defined by the Bragg angle $\Theta_{B}$ is needed. This alignment was realized by rotating the sample around a vertical axis (named hereafter TAL axis) driven by a remote controlled step motor through a one-stage worm gear-tooth wheel system having a sensitivity of $0.00225^{\circ}$.
In addition to the exit angle alignment the target holder was designed to permit two further movements of the sample: a vertical translation along the TAL axis and a rotation $\vartheta$ around an axis normal to the sample surface. For both the translation and rotation two piezo motors were used. The piezo motor ensuring the sample rotation was installed on a support mounted itself on the piezo motor controlling the translation. The samples were glued on intermediate aluminium plates which were screwed on the piezo motor used for the rotation. For each sample the thickness of the plate was chosen so that the TAL axis passed through the sample surface.

\section{Results}

\subsection{Cr pads}

The first investigated periodic structure consisted of 2250 chromium cylinders with a diameter of $2.7 \mu \mathrm{m}$ and a height of $50 \mathrm{~nm}$. The cylinders were arranged in 7 lines along the wafer surface and the minimal distance between two adjacent cylinders was $50 \mu \mathrm{m}$ resulting in a surface coverage by the $\mathrm{Cr}$ structure of about $0.2 \%$.

The pads' pattern was first imprinted into a photoresist by electron beam lithography. Then the whole surface was covered with a $50 \mathrm{~nm}$ thick layer of chromium. After removal of the photoresist, the structure defined by the lithography remained on the surface.

The GIXRF experiment was set up at the FCM beamline of PTB. The sample was mounted so that the pads' rows were aligned along the direction defined by the beam footprint [32].

\subsubsection{Angular profile features}

The characteristic peaks related to the periodicity of the sample could not be observed in the measured GIXRF angular profiles. Actually only features corresponding to particle like structures were seen.

Nevertheless, the spectra show some characteristics of dense particle distributions that can be reproduced only with the GO model [5]. For instance the slope of the intensity growth at shallow angles clearly corresponds to the shadowing effect of consecutive pads. Similarly the intensity attenuation of the beam passing through the relatively long pads is reflected by the intensity decrease around the critical angle. As shown in Fig. 6 these effects are well reproduced by the GO simulation.

\subsection{Cr stripes}

A further aim of our investigations was to probe the effect of a varying period on the angular profiles. For that purpose a sample with periodic chromium stripes was prepared. GEXRF and GIXRF measurements were performed. To vary the period of the pattern keeping the surface coverage constant, the fluorescence intensity was collected at different orientations of the stripes with respect to the vertical axis, i.e., for different angles $\vartheta$. 


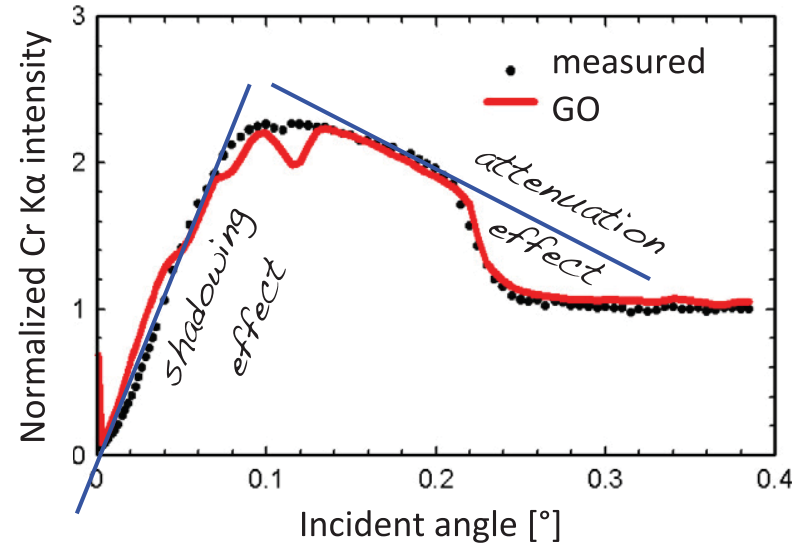

Fig. 6. GIXRF angular profile of the $50 \mathrm{~nm}$ high chromium pads measured with an incident photon energy of $8040 \mathrm{eV}$. Black dots: measured data; red line: GO simulation including the effect of the surface roughness ( $5 \mathrm{~nm}$ rms). The GO calculations correctly reproduce the shadowing and attenuation effects (shown with the blue trend lines). Experimental and theoretical data were normalized to 1 at the largest incidence angle.

The sample consisted of a pattern of 1000 parallel $\mathrm{Cr}$ stripes. The latter were $6 \mathrm{~mm}$ long, $l_{0}=1 \mu \mathrm{m}$ wide and $10 \mathrm{~nm}$ high with a periodicity $p_{0}=6 \mu \mathrm{m}$. The pattern was imprinted on a PMMA-coated silicon wafer by means of the electron-beam lithography technique (beam diameter of $20 \mathrm{~nm}$ ). After the photoresist development the surface was covered with a $10 \mathrm{~nm}$ layer of chromium. Finally, the $\mathrm{Cr}$ stripes' pattern was obtained by means of the lift-off technique.

\subsubsection{Angular profile features}

The GEXRF and GIXRF angular profiles of the Cr stripes' sample are represented in Fig. 7. As shown, for $\vartheta=0$, i.e., when the stripes are parallel to the plane of, respectively, incidence (GIXRF) or emission (GEXRF), angular profiles similar to that of a $10 \mathrm{~nm}$ thick $\mathrm{Cr}$ layer are obtained (left top panel). With an increasing $\vartheta$, a peak which is characteristic of particle-like structures becomes visible around the critical angle of the substrate.

These two features are not surprising and they can be reproduced by making some small changes in currently used methods. However, for profiles recorded at higher values of $\vartheta$, intensity modulation peaks are observed above the critical angle of the substrate in addition to the two main features (see right top panel and bottom panels of Fig. 7). A detailed inspection of the profiles, which is presented elsewhere [50], shows that the angular separation between two consecutive modulation peaks decreases with the tilt angle $\vartheta$. This new feature which was not observed before is related to the sample periodicity as shown below.

\subsubsection{Intensity modulation positions}

The angular positions of the intensity modulation maxima can be interpreted as the incidence angles for which multiple reflections occur and hence for which the number of different ray paths reaching the same fluorescence point is maximized. The angular positions can

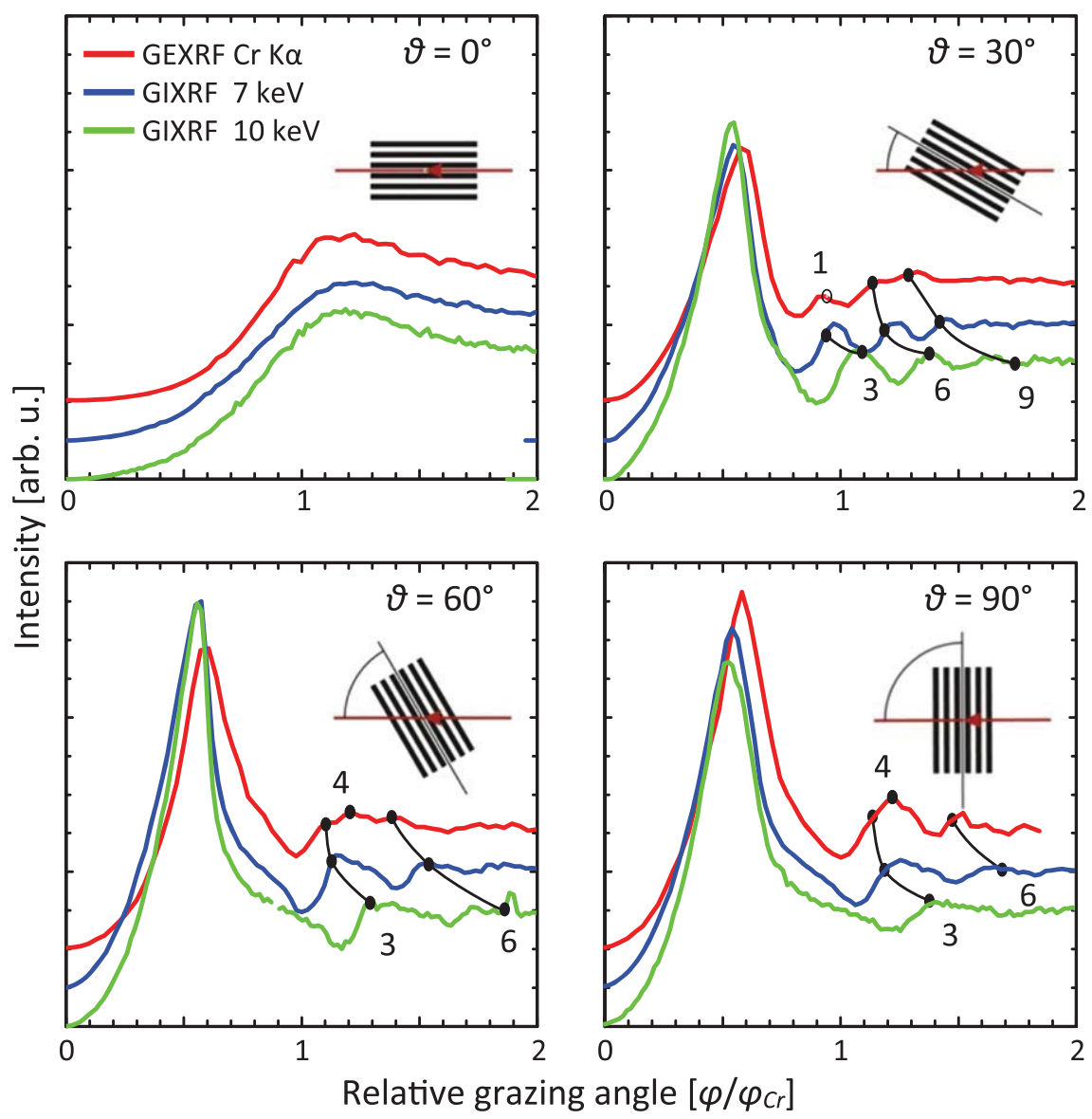

Fig. 7. GEXRF and GIXRF angular profiles of the $\mathrm{Cr}$ stripes' sample measured at different angles $\vartheta$. The sample fluorescence intensity refers to the $\mathrm{Cr}$ K $\alpha \mathrm{X}$-rays which were produced by means of $5.415 \mathrm{keV}$ photons in the GEXRF measurements (red curves) and $7 \mathrm{keV}$ (blue curves) and $10 \mathrm{keV}$ photons (green curves) in the GIXRF measurements. Calculated positions of the modulation peaks are indicated with black dots. Modulation peaks corresponding to the same value of the index $M$ are connected with black lines labeled with the corresponding $M$ value. The GO calculations were performed using tabulated optical constants and an adapted value for the imaginary part of the $\mathrm{Cr}$ refractive index. The grazing angles scales were adjusted to the $\mathrm{Cr}$ critical angle $\varphi_{\mathrm{Cr}}$. 
thus be derived from Eq. (1). Substituting $p$ with $p_{0} / \sin \vartheta$ and using formula (6) the condition for obtaining the modulation maxima can be written as follows:

$$
\tan \varphi_{M}=\frac{M h \sin \vartheta \sin \varphi_{M}}{p_{0} \operatorname{Re}\left(\sqrt{n^{2}-\cos ^{2}\left(\varphi_{M}\right)}\right)}, \quad M \in \mathbb{Q}_{+} .
$$

In other words the positions of the modulation maxima can be calculated using only the refractive index $n_{\mathrm{Cr}}$, height $h$ and period $p_{0}$ of the $\mathrm{Cr}$ structure and the tilt angle $\vartheta$.

However, to reproduce well the measured positions of the modulation maxima with Eq. (7) the imaginary part of the refractive index of $\mathrm{Cr}$ had to be multiplied by a factor $\alpha$. For all GEXRF and GIXRF measurements the factor $\alpha$ for which the best agreement between the experiment and the GO simulations was obtained for the maxima positions was found to be 2.5 . We are inclined to believe that the increased value of the imaginary part of the refractive index reflects the nonconsidered radiation scattering at vertical interfaces. The assumption that the reflectivity of these vertical interfaces is negligibly small may indeed fail due to a nonnegligible surface roughness of the interfaces.

Finally, it should be noted that Eq. (7) gives a necessary condition for the intensity maxima to occur. However, due to more complicated interference effects not all of the calculated maxima are visible in the measured spectra.

\subsection{Cr wedges}

The effects of the structure period on the GEXRF and GIXRF angular profiles having been ascertained, the influence of the lateral dimensions of the pattern elements and thus the structure surface coverage was examined. To this aim, a sample with a $\mathrm{Cr}$ wedge-like pattern was prepared.

A $10.7 \mathrm{~nm}$ chromium layer was deposited on a Si wafer by means of thermal evaporation at room temperature. The layer was then coated with a photoresist and the pattern was imprinted by a laser beam. After development of the photoresist a wet etched $\mathrm{Cr}$ layer was obtained. Finally, the remaining photoresist was removed and a pattern consisting of 500 identical trapezoidal prisms having a length of $2 \mathrm{~cm}$ and widths varying from $2 \mu \mathrm{m}$ to $10 \mu \mathrm{m}$ was obtained. The distance between consecutive wedges, i.e., the period of the structure, was $12 \mu \mathrm{m}$. According to specification the rms roughness of the $\mathrm{Cr}$ surface was within $1 \mathrm{~nm}$.

The sample was measured with the GEXRF technique at various positions of the beam spot on the structure in order to investigate the variation of the GEXRF profiles as a function of the wedge width while keeping the structure period fixed. All measurements were performed with a perpendicular wedge orientation with respect to the detection direction, resulting in a propagation of the fluorescence photons across multiple wedges.

\subsubsection{Angular profile features}

The obtained GEXRF angular profiles (see left panel of Fig. 8) evolve from particle-like profiles for the narrowest structures to layer-like profiles for the widest structures. However, the measured angular dependencies of the fluorescence intensity are far from what one could expect for a single wedge or a continuous layer. In the first case the particle-like profile exhibits indeed a strong shadowing effect, whereas the layer-like profile presents an inflection point at the critical angle of the substrate $\varphi_{\mathrm{Si}}$ and not at the critical angle $\varphi_{\mathrm{Cr}}$ as one would expect for a continuous $\mathrm{Cr}$ layer.

The angular profiles demonstrate a strong dependence on the surface coverage. The most visible changes concern the angular region lying between $\varphi_{\mathrm{Si}}$ and $\varphi_{\mathrm{Cr}}$. At $\varphi_{\mathrm{Si}}$ a peak which is characteristic of particle-like structures appears. Around $\varphi_{\mathrm{Cr}}$ the intensity increases again creating an intensity valley between the two critical angles. One sees that the depth of the valley decreases with the surface coverage. For the widest wedges the particle-like peak is no more visible but the GEXRF intensity below $\varphi_{\mathrm{Cr}}$ is still bigger than the one calculated for a continuous layer.

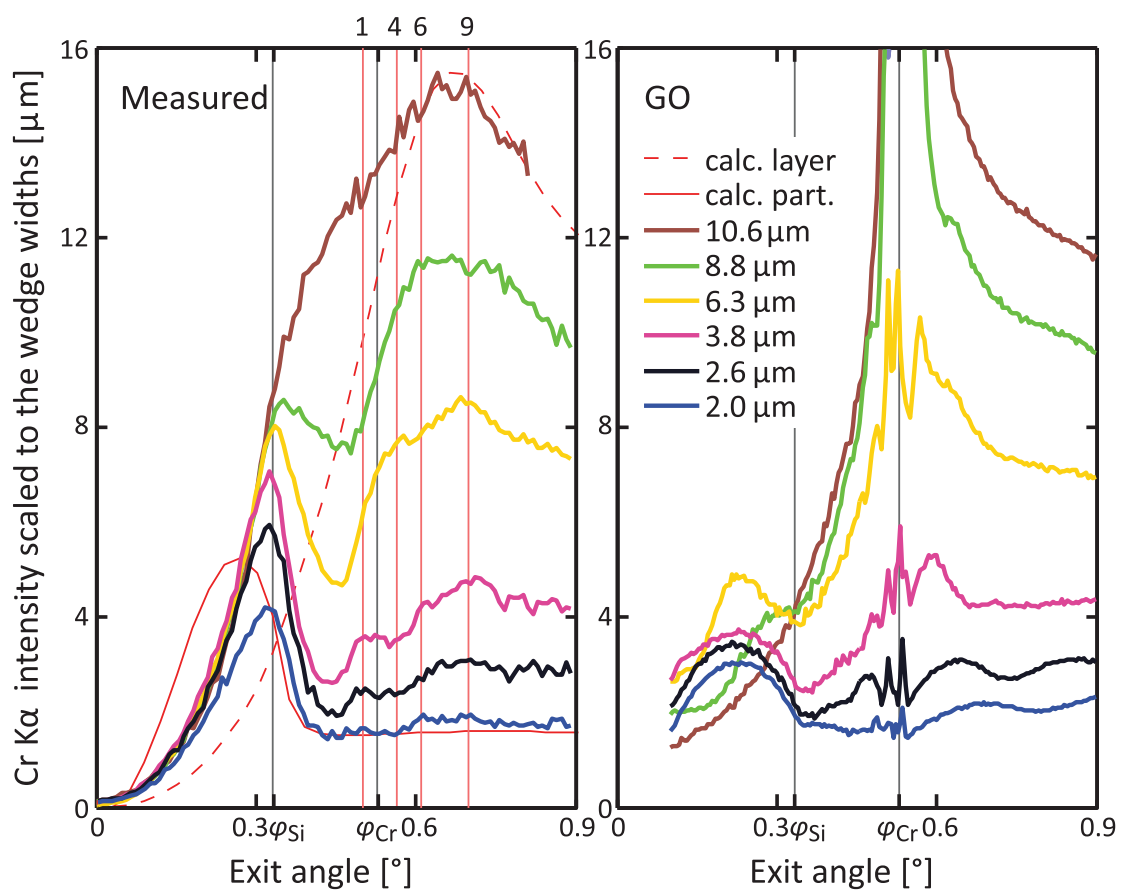

Fig. 8. GEXRF angular profiles of the $\mathrm{Cr}$ wedges' sample for different beam positions along the wedge length axis. Corresponding wedge widths are indicated in the legend. In the left panel the measured data are presented together with the profiles obtained from the $\mathrm{GO}$ model for a single $10.7 \mathrm{~nm}$ high particle (red solid line) and a $10.7 \mathrm{~nm}$ continuous $\mathrm{Cr}$ layer (red dashed line). The right panel shows the results of the GO calculations which were performed assuming a $1 \mathrm{~nm}$ rms roughness for the wedges' surface. Corresponding theoretical and experimental profiles are depicted with the same color. The vertical red lines (left panel) stand for the positions of the intensity modulation maxima labeled with the index $M$ and the gray lines for the critical angles for $\mathrm{Si}$ and $\mathrm{Cr}$. The fluorescence intensity was normalized to account for the differences in the surface coverage. 
Effects of multiple reflections can also be seen. The vertical red lines in the left panel of Fig. 8 represent the positions of the intensity modulation maxima calculated with the modified refractive index (see Section 5.2.2). The intensity modulation maxima with the same indices $M$ as those observed with the $\mathrm{Cr}$ stripes' sample are visible. Due to the interplay of constructive and destructive interferences the intensity bumps appear and are blurred out as a function of the wedge width. This is the effect that was also observed in test simulations prior to experiments (see Section 1).

\subsubsection{GO calculations}

Owing to simple geometrical properties of the $\mathrm{Cr}$ wedges (constant height and only horizontal and vertical interfaces), a limited number of ray paths were required for the calculations. Indeed, for a given X-ray radiation direction, the refraction at the chromium surface can be realized only in two ways, namely at the horizontal and vertical interfaces. Thus, for a given fluorescence point source only four final ray paths had to be considered: two ray paths (up and down) for the X-ray radiation refracted at the horizontal interfaces, and two ray paths (up and down) for the X-ray radiation refracted at the vertical interfaces. Consecutive ray paths arise due to the multiple reflections at the horizontal interfaces of the chromium wedge. As in the simulations the ray paths are computed backwards, i.e., in the inverse direction of the X-ray propagation, one can state that a given fluorescence point can be reached after $0,1,2$, etc. reflections (see Fig. 9).

In order to better reproduce the experiment a $\mathrm{Cr}$ surface rms roughness of $1 \mathrm{~nm}$ was introduced in the simulations whose results are presented in the right panel of Fig. 8. One sees that the GO ray tracing method reproduces both the particle-like and layer-like features. In addition, above the critical angle for the substrate $\varphi_{\mathrm{S} \text {, }}$, a variation of the fluorescence intensity can be clearly distinguished. As for the $\mathrm{Cr}$ stripes' sample, this variation can be assumed to reflect the modulation of the GEXRF intensity as a result of multiple reflections.

Even though the GO simulations do not reproduce the measured data the main trends are predicted correctly. In particular, one can observe a very similar evolution of the intensity valley between the critical angles $\varphi_{\mathrm{Si}}$ and $\varphi_{\mathrm{Cr}}$. The variation of the intensity modulation due to multiple reflections is also similar. However, the intensity modulation maxima appear in this case at the angular positions corresponding to the unmodified refractive index.

In the present simulations the emitted radiation was considered to be fully coherent. As a consequence the calculated constructive interferences resulting from multiple reflections were overrated. This intensity overestimation is the most visible for the largest wedge widths for which the simulations differ to a large extent from the experimental data, especially around $\varphi_{\mathrm{Cr}}$ where sky-rocketing growths of the peaks occur in the simulated profiles.

The simulated intensity evolution below $\varphi_{\mathrm{Si}}$ does not agree with the experimental data either. The shadowing effect due to the traveling of

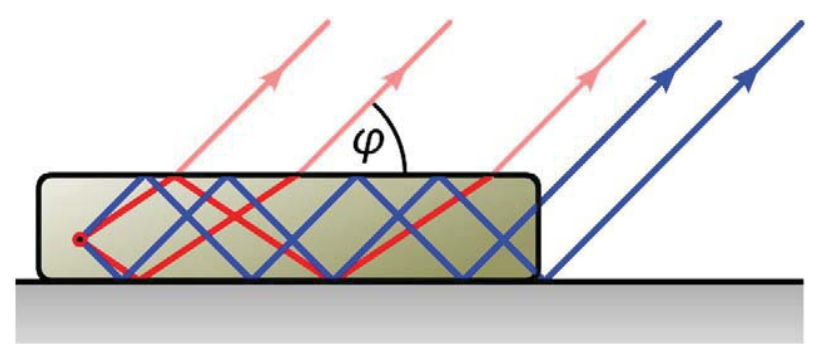

Fig. 9. Various ray paths required for the GO calculations. In the inverse direction of propagation employed in the simulations, the red lines stand for the ray paths reaching the fluorescence point from the horizontal top interface and the blue lines represent the ray paths reaching the fluorescence point from the vertical interface. As can be seen only four final ray path directions (two up and two down) are to be considered.
$\mathrm{X}$-rays through consecutive wedges is not well reproduced. The same holds for the particle-like peak which is shifted to lower exit angles and lies thus closer to the peak corresponding to a full particle-like structure. This might be due to an underestimation of the Si surface roughness. The latter indeed suppresses the coherent reflection of $\mathrm{X}$-rays, which results in an enhancement of the particle-like character of the measured intensity distribution.

\subsection{Disks' patterns}

The $\mathrm{Cr}$ stripes' and $\mathrm{Cr}$ wedges' samples discussed above were characterized by a one-dimensional periodicity or a line symmetry. It is thus quite natural that our investigations were extended to surface structure having a two-dimensional periodicity or plane symmetry. In this last step of our study, multi-elemental samples were also analyzed.

The samples were prepared using the so-called stencil lithography method [51] which is a high resolution shadow-mask technique. A stencil, i.e., a membrane with customized apertures, is clamped to a substrate. The clamped set is placed in an evaporator and the chosen material is then deposited through the stencil's apertures onto the substrate. Stencils with four $800 \times 800 \mu^{2}$ membranes were used. Circular holes with a diameter $d$ of 1-3 $\mu$ m were drilled in the membranes to form $\mathrm{p} 4 \mathrm{~m}$ symmetry [52] periodic patterns with a different hole spacing $a$ for each membrane. For the present project the stencil membranes were placed on a $\mathrm{SiO}_{2}$ substrate.

Two types of disk structures were prepared. The first one was made by depositing $5 \mathrm{~nm}$ thick $\mathrm{Cr}$ disks. For the second one, the deposited pattern consisted of disks made of Co layers superposed to Ni layers, each layer having a thickness of $3 \mathrm{~nm}$. AFM measurements showed that the rms roughness of these samples was of the order of $0.5 \mathrm{~nm}$.

Only high-resolution GEXRF measurements were performed. The data were collected for different orientations of the sample surface, i.e., for different angles $\vartheta$ corresponding to different periodicities of the sample.

\subsubsection{Angular profile features}

As expected intensity modulations were also observed in the profiles of the disks' pattern samples (see Fig. 10). Moreover, their positions and intensity change significantly with the periodicity, i.e., the disk spacing $a$ and angle $\vartheta$.

Since $\mathrm{Co}$ and Ni have very similar refractive indices for both the Co and $\mathrm{Ni} \mathrm{K} \alpha$ fluorescence lines the refraction and reflection at the $\mathrm{Co} / \mathrm{Ni}$ interface are very small and can be neglected. As a consequence, the $\mathrm{Co}$ and Ni GEXRF signals from each $\mathrm{Co} / \mathrm{Ni}$ disk can be regarded as signals from the top and bottom part of an optically uniform disk.

Looking at the GEXRF profiles of the Co/Ni disks' sample (Fig. 11) one can see that the first intensity peak appears at a different position than the one corresponding to the critical angles of the substrate $\left(\varphi_{\mathrm{Si}}\right)$ and disks' materials $\left(\varphi_{\mathrm{Co}}\right.$ and $\left.\varphi_{\mathrm{Ni}}\right)$. In fact, the fluorescence signal from the top Co layer exhibits an intensity growth at $\varphi_{\mathrm{Si}}$, which corresponds to a particle-like signal, whereas the fluorescence intensity from the bottom Ni layer grows at $\varphi_{\mathrm{Co}} / \varphi_{\mathrm{Ni}}$, which indicates a layer-like character of the structure. These observations lead to the conclusion that the particle-like part of the profile is due only to the top parts of the $\mathrm{Co} / \mathrm{Ni}$ disks.

In addition, the intensity modulation induced by the multiple reflections is again visible in most spectra. Here the change of the position of the modulation intensity maxima with the angle $\vartheta$ is also obvious. The two profiles measured at $\vartheta=45^{\circ}$ exhibit another important feature. They both show a structure around $\varphi_{\mathrm{Co}}$ and $\varphi_{\mathrm{Ni}}$; however, for the Co profile (top layer) an intensity dip is observed, whereas for the $\mathrm{Ni}$ profile (bottom layer) a local maximum of intensity is found. This explicitly reflects the influence of different ray paths on the photon interferences. 

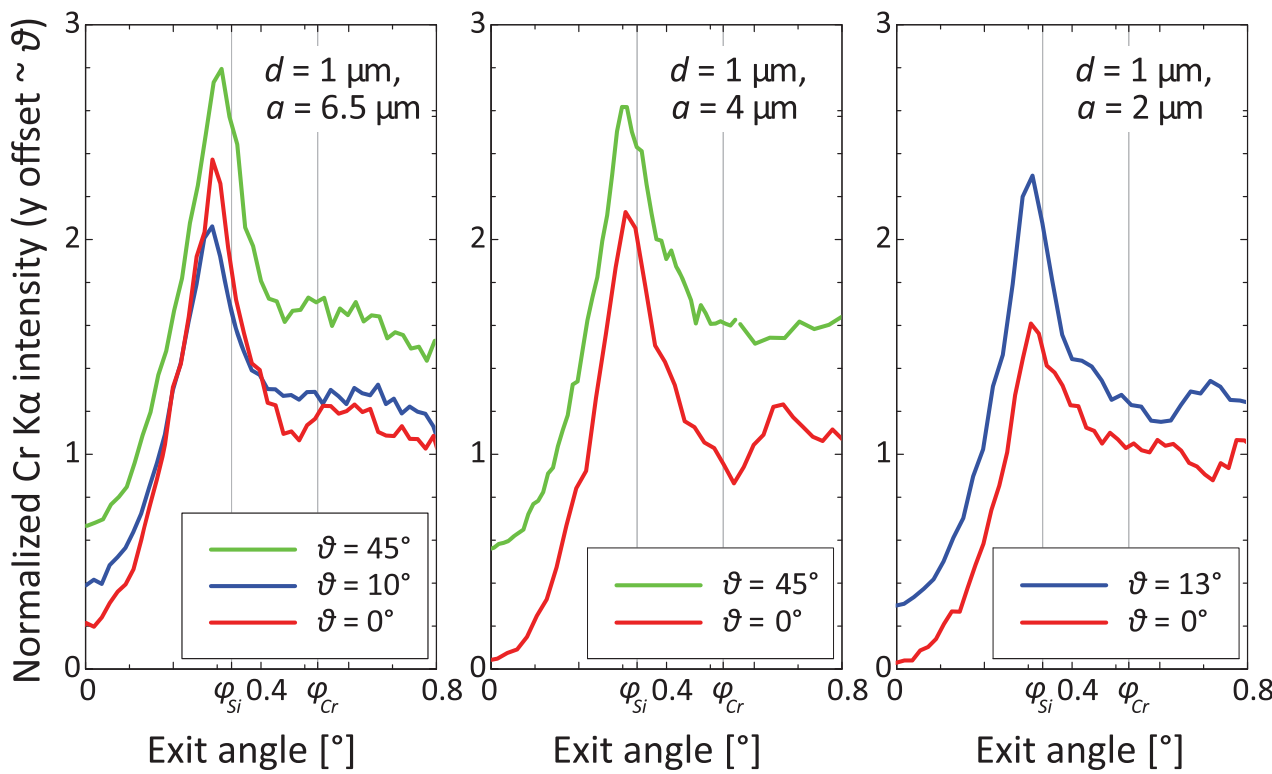

Fig. 10. GEXRF profiles of p4m Cr disks' patterns. The diameter $d$ of the disks is the same for the three samples but the period $a$ is different. Each sample was measured for at least two different angles $\vartheta$.

\section{Conclusions}

Nano-sized periodic surface structures were investigated by means of the GIXRF and high-resolution GEXRF methods. The sample fluorescence was measured as a function of the grazing incidence (GIXRF) or grazing exit (GEXRF) angle. The so-obtained angular intensity profiles were compared to simulations based on GO calculations.

The present study shows that the GEXRF and GIXRF methods are very sensitive to the periodicity, surface coverage and size of the structure elements and that they therefore represent powerful tools for the morphology characterization of nano-sized surface structures. This work demonstrates also that the GO calculations reproduce quite well the main features of the experimental XRF intensity profiles. In the latter features that are characteristic of particle-like and layer-like structures were observed. Additional features related to the periodicity of the structures could also be seen. These additional features were found to strongly depend on the specific characteristics of the pattern.

Patterns consisting of small and well separated elements present a clear particle-like evolution of the XRF intensity with the grazing angle. An increase of the lateral dimensions of the elements leads to the attenuation of the particle-like signal in the region close to the critical angle of the substrate material and to the appearance of a layer-like component.

For dense particle distributions additional effects which are due to the propagation of the X-rays through several neighboring elements of the structure occur. Two types of such effects were observed. First, for small grazing angles, the shadowing of consecutive structure elements leads to the attenuation of the intensity in the particle-like XRF signal. This effect is more pronounced for dense patterns and high structure elements. The second effect is related to the fact that the X-ray radiation
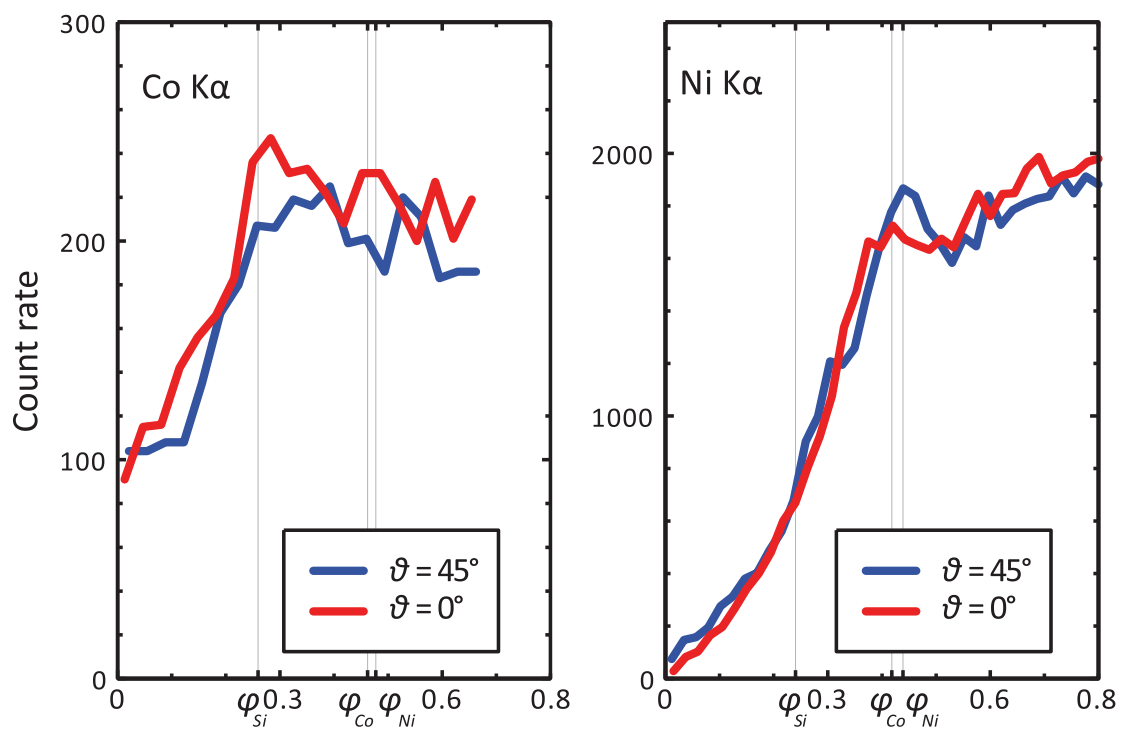

Exit angle [ $\left.{ }^{\circ}\right]$

Exit angle $\left[{ }^{\circ}\right]$

Fig. 11. Co K $\alpha$ (left panel) and Ni K $\alpha$ (right panel) GEXRF profiles of the Co/Ni disk' pattern ( $d=3 \mu \mathrm{m}, a=6 \mu \mathrm{m}$ ) for two different orientations of the sample surface. Co K $\alpha$ and Ni K $\alpha$ signals correspond to the top, and the bottom layer respectively. 
can be reflected many times in consecutive elements of the structure. As a consequence, for a given fluorescence point and certain grazing angles the number of ray paths contributing to the XRF signal is significantly higher with respect to a particle-like structure. For periodic patterns this effect is reflected in the angular profile by the occurrence of a nearly-periodic variation of the XRF intensity. According to GO simulations, the relative maxima of these intensity modulations appear at the angles corresponding to the biggest numbers of ray paths. In principle from the positions of the intensity modulation peaks the height and period of the pattern might be retrieved. In addition, provided that the reasoning presented in Section 5.2.2 is correct, the adapted value for the imaginary part of the refractive index employed in calculations may be a measure of the surface roughness of vertical interfaces.

\section{Acknowledgments}

We would like to acknowledge the assistance of Dr. Ivan Marozau from the University of Fribourg for the AFM measurements. We are also grateful to the staffs of the ID21 beamline of the ESRF, SuperXas beamline of the SLS and four-crystal monochromator beamline of BESSY II for providing our experiments with photon beams of excellent quality. The financial supports of the Swiss National Science Foundation (project number 200020_135040) and the ESRF (experiment MI-1108) are acknowledged. Two of us (P.H. and F.R.) thank the Investitionsbank in Berlin for the financial support of the ProFIT/EFRE project 'Nanoparticle X-ray Analytics'.

\section{References}

[1] D.K.G. de Boer, Influence of the roughness profile on the specular reflectivity of $X$ rays and neutrons, Phys. Rev. B 49 (1994) 5817-5820.

[2] K. Tsuji, T. Yamada, T. Utaka, K. Hirokawa, The effects of surface roughness on the angle-dependent total-reflection X-ray fluorescence of ultrathin films, J. Appl. Phys. 78 (1995) 969-973.

[3] D. Windt, IMD-software for modeling the optical properties of multilayer films, Comput. Phys. 12 (1998) 360

[4] M.K. Tiwari, G.S. Lodha, K.J.S. Sawhney, Applications of the 'CATGIXRF' computer program to the grazing incidence X-ray fluorescence and X-ray reflectivity characterization of thin films and surfaces, X-Ray Spectrom. 39 (2010) 127-134.

[5] S.H. Nowak, F. Reinhardt, B. Beckhoff, J.-Cl. Dousse, J. Szlachetko, Geometrical optics modelling of grazing incidence X-ray fluorescence of nanoscaled objects, J. Anal. At Spectrom. 28 (2013) 689-696.

[6] A. Kubala-Kukuś, D. Banaś, W. Cao, J.-Cl. Dousse, J. Hoszowska, Y. Kayser, M. Pajek, M. Salomé, J. Susini, J. Szlachetko, M. Szlachetko, Observation of ultralow-level Al impurities on a silicon surface by high-resolution grazing emission X-ray fluorescence excited by synchrotron radiation, Phys. Rev. B 80 (4) (2009) 113305.

[7] Y. Kayser, J. Szlachetko, D. BanaÅ, W. Cao, J-Cl. Dousse, J. Hoszowska, A. KubalaKukuÅ, M. Pajek, High-energy-resolution grazing emission X-ray fluorescence applied to the characterization of thin Al films on Si, Spectrochim. Acta Part B 88 (2013) 136-149.

[8] A. Bekshaev, R. Van Grieken, Substrates with a periodic surface structure in grazingexit X-ray microanalysis, Spectrochim. Acta Part B 57 (2002) 865-882.

[9] R. Jenkins, X-ray Fluorescence Spectrometry, 2nd ed. Wiley, New York, 1999.

[10] R. Klockenkämper, Total-reflection X-ray Fluorescence Analysis (Chemical Analysis: A Series of Monographs on Analytical Chemistry and Its Applications), WileyInterscience, 1996.

[11] A. von Bohlen, Total reflection X-ray fluorescence and grazing incidence X-ray spectrometry - tools for micro- and surface analysis. A review, Spectrochim. Acta Part B 64 (2009) 821-832.

[12] K. Stoev, K. Sakurai, Review on grazing incidence X-ray spectrometry and reflectometry, Spectrochim. Acta Part B 54 (1999) 41-82.

[13] P.K. de Bokx, Chr. Kok, A. Bailleul, G. Wiener, H.P. Urbach, Grazing-emission X-ray fluorescence spectrometry: principles and applications, Spectrochim. Acta Part B 52 (1997) 829-840.

[14] M. Claes, P. de Bokx, R. Van Grieken, Progress in laboratory grazing emission X-ray fluorescence spectrometry, X-Ray Spectrom. 28 (1999) 224-229.

[15] M. Claes, R. Van Grieken, P. de Bokx, Comparison of grazing emission XRF with total reflection XRF and other X-ray emission techniques, X-Ray Spectrom. 26 (1997) 153-158.

[16] J. Szlachetko, D. Banaś, A. Kubala-Kukuś, M. Pajek, W. Cao, J.-Cl. Dousse, J. Hoszowska, Y. Kayser, M. Szlachetko, M. Kavčič, M. Salomé, J. Susini, Application of the high-resolution grazing-emission X-ray fluorescence method for impurities control in semiconductor nanotechnology, J. Appl. Phys. 105 (2009) 086101.

[17] U. Weisbrod, R. Gutschke, J. Knoth, H. Schwenke, Total reflection X-ray fluorescence spectrometry for quantitative surface and layer analysis, Appl. Phys. A 53 (1991) 449-456.
[18] Y. Kayser, D. Banaś, W. Cao, J.-Cl. Dousse, J. Hoszowska, P. Jagodziński, M. Kavčič, A. Kubala-Kukuś, S. Nowak, M. Pajek, J. Szlachetko, Depth profiles of Al impurities implanted in Si wafers determined by means of the high-resolution grazing emission X-ray fluorescence technique, Spectrochim. Acta Part B 65 (2010) 445-449.

[19] Y. Kayser, D. Banaś, W. Cao, J.-Cl. Dousse, J. Hoszowska, P. Jagodziński, M. Kavčič, A. Kubala-Kukuś, S. Nowak, M. Pajek, J. Szlachetko, Depth profiling of dopants implanted in Si using the synchrotron radiation based high-resolution grazing emission technique, X-Ray Spectrom. 41 (2012) 98-104

[20] P. Hönicke, Y. Kayser, B. Beckhoff, M. Müller, J.-Cl. Dousse, J. Hoszowska, S.H. Nowak, Characterization of ultra shallow aluminum implants in silicon by grazing incidence and grazing emission X-ray fluorescence spectroscopy, J. Anal. At. Spectrom. 27 (2012) 1432-1438

[21] A. Bekshaev, R. Van Grieken, Interference technique in grazing-emission electron probe microanalysis of submicrometer particles, Spectrochim. Acta Part B 56 (2001) 503-515.

[22] J. Osán, F. Reinhardt, B. Beckhoff, A.E. Pap, S. Török, Probing patterned wafer structures by means of grazing incidence X-ray fluorescence analysis, ECS Trans. 25 (2009) 441-451

[23] M.K. Tiwari, G.M. Bhalerao, M. Babu, A.K. Sinha, C. Mukherjee, Investigation of metal nanoparticles on a Si surface using an X-ray standing wave field, J. Appl. Phys. 103 (2008) 054311

[24] A. von Bohlen, M. Krämer, C. Sternemann, M. Paulus, The influence of X-ray coherence length on TXRF and XSW and the characterization of nanoparticles observed under grazing incidence of X-rays, J. Anal. At. Spectrom. 24 (2009) 792.

[25] H. Schwenke, P. A. Beaven, J. Knoth, Total reflection and grazing emission X-ray fluorescence spectrometry: Assessment of the size of contaminant particles on silicon wafer surfaces, in: K. L. Mittal (Ed.), Particles on Surfaces 7: Detection, Adhesion and Removal., pp. 11-26.

[26] H. Schwenke, J. Knoth, U. Weisbrod, Analysis of particles on surfaces by total reflection X-ray fluorescence spectrometry, Particles on Surfaces 3: Detection, Adhesion, and Removal, Springer, 1991, pp. 131-141.

[27] B. Schneider, The determination of atmospheric trace metal concentrations by collection of aerosol particles on sample holders for total-reflection X-ray fluorescence, Spectrochim. Acta Part B 44 (1989) 519-523.

[28] J. Osán, S. Török, B. Alföldy, A. Alsecz, G. Falkenberg, S.Y. Baik, R. Van Grieken, Comparison of sediment pollution in the rivers of the Hungarian Upper Tisza Region using non-destructive analytical techniques, Spectrochim. Acta Part B 62 (2007) 123-136.

[29] S. Pahlke, L. Fabry, L. Kotz, C. Mantler, T. Ehmann, Determination of ultra trace contaminants on silicon wafer surfaces using total-reflection X-ray fluorescence TXRF ‘state-of-the-art', Spectrochim. Acta Part B 56 (2001) 2261-2274.

[30] D.K.G. de Boer, A.J.G. Leenaers, W.W. van den Hoogenhof, Glancing-incidence X-ray analysis of thin-layered materials: a review, X-Ray Spectrom. 24 (1995) 91-102.

[31] P. Hönicke, B. Beckhoff, M. Kolbe, D. Giubertoni, J. Berg, G. Pepponi, Depth profile characterization of ultra shallow junction implants, Anal. Bioanal. Chem. 396 (2010) 2825-2832.

[32] F. Reinhardt, J. Osán, S. Török, A.E. Pap, M. Kolbe, B. Beckhoff, Reference-free quantification of particle-like surface contaminations by grazing incidence X-ray fluorescence analysis, J. Anal. At. Spectrom. 27 (2012) 248-255.

[33] R.S. Becker, J.A. Golovchenko, J.R. Patel, X-ray evanescent-wave absorption and emission, Phys. Rev. Lett. 50 (1983) 153-156.

[34] D.K.G. de Boer, Glancing-incidence X-ray fluorescence of layered materials, Phys. Rev. B 44 (1991) 498-511.

[35] H.P. Urbach, P.K. de Bokx, Calculation of intensities in grazing-emission X-ray fluorescence, Phys. Rev. B 53 (1996) 3752-3763.

[36] R. Carminati, M. Nieto-Vesperinas, J.-J. Greffet, Reciprocity of evanescent electromagnetic waves, J. Opt. Soc. Am. A 15 (1998) 706-712.

[37] T. Noma, A. Iida, K. Sakurai, Fluorescent-X-ray-interference effect in layered materials, Phys. Rev. B 48 (1993) 17524.

[38] P. Skytt, B. Gălnander, T. Nyberg, J. Nordgren, P. Isberg, Probe depth variation in grazing exit soft-X-ray emission spectroscopy, Nucl. Inst. Methods Phys. Res. A 384 (1997) 558-562.

[39] P. De Bokx, H. Urbach, Laboratory grazing-emission X-ray fluorescence spectrometer, Rev. Sci. Instrum. 66 (1995) 15-19.

[40] J. Szlachetko, J.-Cl. Dousse, J. Hoszowska, M. Berset, W. Cao, M. Szlachetko, M. Kavčič, Relative detection efficiency of back-and front-illuminated chargecoupled device cameras for X-rays between $1 \mathrm{keV}$ and $18 \mathrm{keV}$, Rev. Sci. Instrum. 78 (2007) 093102

[41] L. Nevot, B. Pardo, J. Corno, Characterization of X-UV multilayers by grazing incidence X-ray reflectometry, Rev. Phys. Appl. 23 (1988) 1675-1686.

[42] H. Schwenke, R. Gutschke, J. Knoth, M. Kock, Treatment of roughness and concentration gradients in total reflection X-ray fluorescence analysis of surfaces, Appl. Phys. A 54 (1992) 460-465.

[43] K. Tsuji, S. Sato, K. Hirokawa, Glancing-incidence and glancing-takeoff X-ray fluorescence analysis of a Mn ultrathin film on an Au layer, Thin Solid Films 274 (1996) $18-22$.

[44] C. Horntrich, F. Meirer, C. Streli, P. Kregsamer, G. Pepponi, N. Zoeger, P. Wobrauschek, Influence of the sample morphology on total reflection X-ray fluorescence analysis, Powder Diffract. 24 (2009) 140-144.

[45] U.E.A. Fittschen, M. Menzel, O. Scharf, M. Radke, U. Reinholz, G. Buzanich, V. Montoya, K. McIntosh, C. Streli, G.J. Havrilla, Observation of X-ray shadings in SRTXRF using a colour X-ray camera (CXC), Spectrochim. Acta Part B (2014) (forthcoming).

[46] B. Beckhoff, A. Gottwald, R. Klein, M. Krumrey, R. Müller, M. Richter, F. Scholze, R. Thornagel, G. Ulm, A quarter-century of metrology using synchrotron radiation by PTB in Berlin, Phys. Status Solidi B 246 (2009) 1415-1434. 
[47] J. Lubeck, B. Beckhoff, R. Fliegauf, I. Holfelder, P. Hönicke, M. Müller, J. Weser, Development of a new instrumentation for reference-free nanoanalytics by means of X-ray spectrometry, Rev. Sci. Instrum. 84 (2013) 045106.

[48] B. Beckhoff, Reference-free X-ray spectrometry based on metrology using synchrotron radiation, J. Anal. At. Spectrom. 23 (2008) 845-853.

[49] J. Hoszowska, J.-Cl. Dousse, J. Kern, C. Rhême, High-resolution von Hamos crystal X-ray spectrometer, Nucl. Inst. Methods Phys. Res. A 376 (1996) 129-138.

[50] F. Reinhardt, S. Nowak, B. Beckhoff, J.-Cl. Dousse, M. Schoengen, Grazing Incidence X-ray Fluorescence of Periodic Structures - A Comparison Between X-ray Standing
Waves and Geometrical Optics Calculations, to be published in J. Anal. At Spectrom (2014).

[51] G. Villanueva, O. Vazquez-Mena, M. van den Boogaart, K. Sidler, K. Pataky, V. Savu, J. Brugger, Etching of sub-micrometer structures through Stencil, Microelectron. Eng. 85 (2008) 1010-1014

[52] H. Wondratschek, Classifications of space groups, point groups and lattices, in: T. Hahn (Ed.), 5 edition, International Tables for Crystallography, Volume A: Spacegroup SymmetryInternational Union of Crystallography, Chester, England, 2006, pp. 726-731. 